\title{
Coronal structure geometries on pre-main sequence stars
}

\author{
F. Favata ${ }^{1}$, G. Micela ${ }^{2}$, and F. Reale ${ }^{3}$ \\ 1 Astrophysics Division - Space Science Department of ESA, ESTEC, Postbus 299, 2200 AG Noordwijk, \\ The Netherlands \\ 2 Osservatorio Astronomico di Palermo, Piazza del Parlamento 1, 90134 Palermo, Italy \\ 3 Dip. Scienze FF. \& AA., Sez. Astronomia, Univ. Palermo, Piazza del Parlamento 1, 90134 Palermo, Italy
}

Received 15 January 2001 / Accepted 21 March 2001

\begin{abstract}
Using a hydrodynamic model we have re-analyzed large flaring events on three different categories of pre-main sequence (PMS) stars: the young stellar object (YSO) YLW 15, the classical T Tauri star (CTTS) LkH $\alpha$ 92, the weak-line T Tauri star (WTTS) V773 Tau, and the WTTS HD 283572 (the first three objects were observed by ASCA, the last by ROSAT; all observations have been previously reported in the literature). The first three flares were previously analyzed on the basis of a quasi-static model mostly used up to now, consistently yielding large loops $\left(L \gtrsim R_{*}\right)$ and no evidence of sustained heating. Our hydrodynamic modeling approach, however, shows that the size of the flaring regions must be much smaller $\left(L \lesssim R_{*}\right)$ and moreover this method shows in all cases evidence of vigorous sustained heating during the flare decay, so that the decay of the observed light curve actually reflects the temporal profile of the heating rather than that of the free decay of the heated loop(s). The events on the protostar YLW 15 have durations comparable to the stellar rotation period, so that their limited size and their lack of self-eclipses give evidence of a polar location on the star. This is in contrast with the recently advanced hypothesis that these flares are due to long loops spanning the region between the star and the accretion disk. In general, the present analysis shows that flaring coronae on PMS stars have a structure similar to the coronae on older active stars.
\end{abstract}

Key words. stars: late-type - activity - coronae; X-rays: stars

\section{Introduction}

Strong X-ray emission is associated with all stages of the early evolution of low-mass stars, from the protostellar Young Stellar Objects - YSO or Class I - to the Classical T Tau - CTTS or Class II - to the Weak-Line T Tau phase - WTTS or Class III. The salient characteristics of stellar activity in the pre-main sequence (PMS) phase have been recently reviewed by Feigelson \& Montmerle (1999): in most cases the observed X-ray emission has been interpreted as due to magnetically confined (and likely magnetically heated) plasma, although with much enhanced - both in absolute and relative terms - activity levels with respect to main-sequence stars of comparable mass. Whether the corona is however just an enhanced version of a "solar-like" one, or whether significantly different mechanisms (and coronal topologies) are at work has been a matter of debate, in particular in relationship to the possible influence of the accretion disk on the corona: in YSO's and CTTS's significant amounts of circumstellar material (mostly in the form of a disk) are present, and magnetic

Send offprint requests to: F. Favata, e-mail: ffavata@astro.esa.int fields are thought to funnel and modulate the accretion onto the (proto)star (e.g. Hartmann 1998). Various possibilities for the magnetic interaction between the star and the disk are for example shown in Figs. 2 and 3 of Feigelson \& Montmerle (1999); understanding the topology and dimensional scales of the coronal plasma (and, indirectly, of the magnetic field) can, in the case of PMS stars, give important constraints to the accretion process. The stellar activity also has a significant influence on the circumstellar environment (see e.g. the review of Glassgold et al. 2000), and for example a low-lying X-ray emitting region will irradiate (and thus ionize) the circumstellar disk much less than a very extended emitting region from which Xrays can reach the disk out to much larger distances from the parent star.

The study of the decay of flares has in the last decade been extensively used to model the characteristics of the flaring regions in different stellar types, and in particular their size. This in turn has been used to derive constraints on the structuring of the active corona, with results which are however, as discussed below, model-dependent. The quasi-static method of flare modeling (originally described by van den Oord \& Mewe 1989) assumes that the flaring 
loops decay freely after an initial impulsive heating event, and that the decay can be described through a series of static states; while in principle the formalism allows for the presence of heating during the decay phase, in practice in no case has the application of the formalism resulted in sustained heating being detected. Thus, in the presence of the long decay times typically observed for large flares in active stars, the quasi-static method consistently yields long loops and extended coronae. This method has been extensively applied in the last decade, resulting in a general assumed framework of coronae of active stars populated with very long, extended loop structures. In the case of PMS stars, these have often been thought to extend to, and link with, the accretion disk, resulting in a distinctly "non-solar" coronal geometry.

More recently, Reale et al. (1997) have developed a method based on hydrodynamic modeling of decaying flaring loops, which is able to detect the presence of heating during the decay and to adjust the estimate of the loop length accordingly, and which has been tested ("calibrated") with good results on solar flares of which the loop length can be checked from images. When applied to the only stellar flare for which an eclipse allows to make a geometric size estimate (the Algol SAX flare, Schmitt \& Favata 1999; Favata \& Schmitt 1999), the quasi-static method has been shown to over-estimate the loop size by a large factor, while the hydrodynamic modeling diagnoses the presence of long-lasting sustained heating during the decay, and yields a more realistic estimate of the region's size (although still overestimating it). Application of this method to a number of events on different types of coronal sources has shown that sustained heating is an essential feature of large stellar flares, and that therefore the flaring structures are actually significantly smaller than so far postulated. This applies to flare stars (CM Leo, Reale \& Micela 1998; EV Lac, Favata et al. 2000; AD Leo, Favata et al. 2000c), to young single solar-type stars (AB Dor, Maggio et al. 2000a), to active binaries (AR Lac and CF Tuc, Favata 2000) and to Algol (Favata \& Schmitt 1999; Favata et al. 2000b).

The hydrodynamic modeling approach is based on detailed simulations of decaying flaring loops. The simulations show that the key diagnostic is the slope $\zeta$ of the flare's decay in the $\log T$ vs. $\log n$ plot (actually, in the case of stellar flares, where no direct density diagnostics are usually available, the $\log T$ vs. $\log \sqrt{E M}^{1}$ plot): if sustained heating is present, the slope will be shallower than in the case of an impulsively heated, freely decaying loop (cf. e.g., Fig. 1). A fit to the parameters of the numerical model allows to derive the length of the flaring loop as a function of the observed decay time scale $\tau_{\mathrm{LC}}$, of the $\zeta$ parameter and of the peak temperature in the flaring loop $T_{\max }$. The latter quantity is defined as the maximum intrinsic temperature of a model loop whose spectrum synthesized in the band of a given X-ray spectrometer yields,

\footnotetext{
1 Where the emission measure is defined as $E M=$ $\int n_{\mathrm{e}} n_{\mathrm{H}} \mathrm{d} V$.
}

when fit with a single-temperature spectrum, a maximum observed temperature $T_{\max }$.

Large flares on PMS stars have in the past always been studied with the quasi-static method, resulting in typical sizes of $L \simeq 10^{11}-10^{12} \mathrm{~cm}$, i.e. $L \geq R_{*}$, which have often led to the hypothesis of loops linking the photosphere with the accretion disk (e.g. Tsuboi et al. 2000). In the present paper we study, through the hydrodynamic modeling method of Reale et al. (1997), stellar X-ray flares observed on PMS stars from Class I to Class III, with the aim of determining whether they feature strong sustained heating (as observed in all stellar types studied so far with this approach) and thus whether the related coronal structures are significantly more compact than postulated up to now. Given the relatively large average distance of starforming regions (and therefore of PMS stars) from Earth, only few (and very intense) X-ray flares from PMS stars have been observed with sufficient statistics, and therefore the choice of the sample studied in this paper is driven by the (random) availability of the data, and is neither complete or unbiased.

The present paper is so structured: Sect. 2 gives a summary description of the sample's characteristics, in Sect. 3 the analysis of each flare is discussed in detail, while in Sect. 4 the results are discussed and summarized.

\section{Sample studied}

We have identified, in the literature, four X-ray flares from PMS's which have sufficient statistics to allow their study with the hydrodynamic modeling approach: a flare observed with ASCA on the protostar YLW 15 (Tsuboi et al. 2000), a flare observed with the ROSAT PSPC on the CTTS LkH $\alpha 92$ (Preibisch et al. 1993), a flare observed with ASCA on the WTTS V773 Tau (Tsuboi et al. 1998) and a PSPC flare on the WTTS HD 283572 (Stelzer et al. 2000). The sample (although small) spans the complete pre-main sequence phase and covers all key evolutionary stages; the somewhat older star AB Dor, just arriving on the main sequence, has been studied with the same approach by Maggio et al. (2000), thus linking the present sample with the subsequent evolutionary stage.

The first three events discussed here have been previously analyzed with the quasi-static method, while the fourth has been reported by Stelzer et al. (2000) but not analyzed in detail. The quasi-static analysis has resulted (as typical for large stellar flares) in large loop sizes $\left(L \simeq 3.5 R_{*}\right.$ for $\mathrm{YLW} 15, L \simeq 0.6 R_{*}$ for $\mathrm{LkH} \alpha 92$, $L \simeq 1.3 R_{*}$ for V773 Tau).

\section{Analysis of the flaring events}

\subsection{YLW 15}

YLW 15 is a low-mass Class I protostar in the $\rho$ Oph starforming region $(d \simeq 165 \mathrm{pc})$, with an estimated radius $R_{*} \simeq 4.2 R_{\odot}=2.9 \times 10^{11} \mathrm{~cm}$ (e.g. Montmerle et al. 2000). Its high $\mathrm{X}$-ray activity was noticed through a large flare 

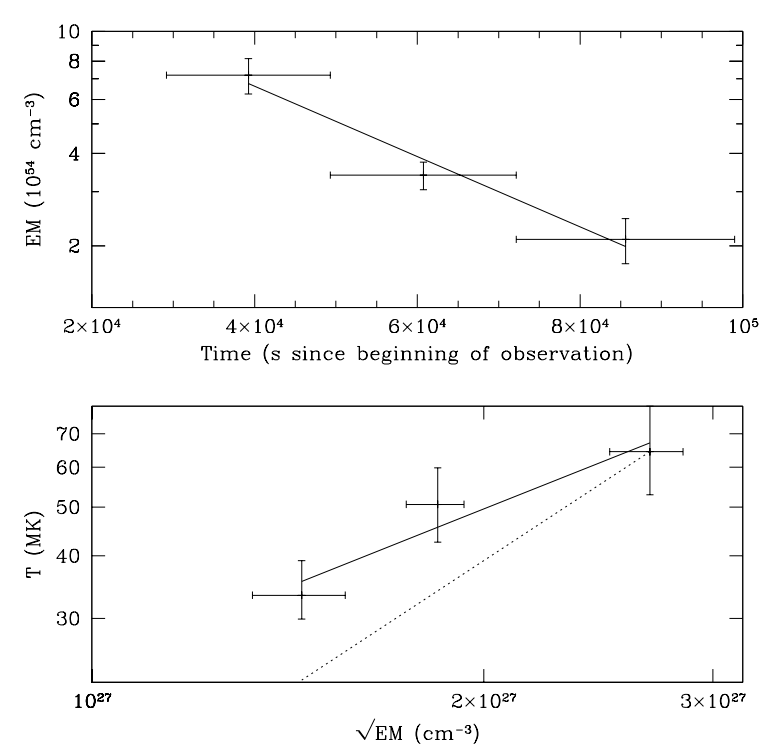

Fig. 1. The evolution of the YLW 15 flare. The top panel shows the evolution of the flare's emission measure (used as a proxy to the light curve, e.g. to the X-ray count rate), together with the best-fit exponential decay, while the bottom panel shows the evolution of the flare's decay in the $\log T$ vs. $\log n$ plane. The solid line is the best fit to the decay $(\zeta=1.0)$, while the dotted line is the path the decay would have followed if it had been quasi-static with no sustained heating during the decay phase $\left(\zeta_{\mathrm{QS}}=1.7\right)$.

observed with the ROSAT HRI (Grosso et al. 1997); the lack of spectroscopic capabilities of the HRI however prevents a detailed analysis of this event. YLW 15 was later observed with ASCA, which detected three consecutive significant flaring events, which are discussed and analyzed by Tsuboi et al. (2000). The first and most intense of the three events has a decay time-scale $\tau_{\mathrm{LC}}=31 \mathrm{ks}$, a measured maximum temperature $T_{\mathrm{d}} \simeq 64 \mathrm{MK}$ and a peak X-ray luminosity of $\simeq 2 \times 10^{32} \mathrm{erg} \mathrm{s}^{-1}$. Tsuboi et al. (2000) have analyzed the event using the quasi-static framework (assuming impulsive heating), deriving a semilength of the flaring loop $L \simeq 10^{12} \mathrm{~cm} \simeq 3.5 R_{*}$, a density $n \simeq 5 \times 10^{10} \mathrm{~cm}^{-3}$ and a minimum value, for the magnetic field, of $B \simeq 150 \mathrm{G}$. Similar physical parameters are derived for the second and third event (which have however lower statistics).

Using the spectral parameters ( $T$ and $E M)$ derived by Tsuboi et al. (2000) for three temporal intervals during the flare decay we have modeled the first flare using the hydrodynamic modeling approach of Reale et al. (1997), recalibrated for use with the ASCA detectors as described by Favata et al. (2000c) and Favata et al. (2000a). Although the flare decay has been divided in only three time intervals (due to the limited number of photons detected), this is sufficient to constrain the presence of sustained heating and thus the loop's length. The flare evolution and its best-fit decay in the $\log T$ vs. $\log n$ plane are shown in Fig. 1. The slope of the decay, $\zeta=1.0 \pm 0.3$ is significantly shallower than the slope that would be observed if the decay were indeed quasi-static $\left(\zeta_{\mathrm{QS}}=1.7\right)$.
The corresponding ratio between the observed decay time and the intrinsic thermodynamic decay cooling time of the loop without additional heating (Serio et al. 1991) is $\tau_{\mathrm{LC}} / \tau_{\mathrm{th}}=F(\zeta) \simeq 2.7[2.2-3.6]^{2}$, indicating the presence of significant sustained heating during the decay. The actual peak temperature of the plasma in the flaring loop is $T_{\max } \simeq 150 \mathrm{MK}$, and the loop length is $L \simeq 4.6[3.5-5.6] \times 10^{11} \mathrm{~cm} \simeq 1.6[1.2-1.9] R_{*}$, a factor of $\simeq 2$ smaller than estimated by the quasi-static method under the assumption of free decay.

To estimate the density $n$ of the plasma and the strength of the confining magnetic field $B$, the volume $V$ of the flaring region and therefore its geometry must be known. Assuming a loop with constant cross-section, whose length is obtained through the flare analysis, the only free parameter is the ratio of the diameter of the loop's cross section to its total length, or the aspect ratio, $\beta$. In the solar context $\beta \simeq 0.1$ is often claimed as "typical", although in the case of active stars higher values (up to $\beta \simeq 0.3$, Maggio et al. 2000; Favata et al. 2000a) have been inferred.

For a semicircular loop, $V=2 \pi L^{3} \beta^{2}$. For the YLW 15 flare we thus derive $V=6.1 \times 10^{33}-5.5 \times 10^{34} \mathrm{~cm}^{3}$ (where the first value is for $\beta=0.1$ and the second for $\beta=0.3$, as in the following). The density of the flaring plasma at the peak of the event can then be derived as $n_{\mathrm{e}}=\sqrt{E M /(0.8 V)}$, where the factor 0.8 is the scaling factor between the number of ions and the number of electrons for a solar $\mathrm{H} / \mathrm{He}$ ratio. This assumes a single loop uniformly filled with plasma. If the loop is significantly filamented (i.e. has a filling factor smaller than 1) the resulting density would be a lower limit to the actual value. In the present case (using the peak $E M=$ $7.2 \times 10^{54} \mathrm{~cm}^{-3}$ reported by Tsuboi et al. 2000), we derive $n_{\mathrm{e}}=3.8 \times 10^{10}-1.3 \times 10^{10} \mathrm{~cm}^{-3}$. The plasma pressure can then be estimated through $p_{\mathrm{e}}=2 n_{\mathrm{e}} k T_{\max }=1600$ 500 dyne $\mathrm{cm}^{-2}$. The equilibrium pressure estimated from the peak temperature and the derived length of the flaring loop through the scaling laws of Rosner et al. (1978) is $p_{\mathrm{RTV}}=2700$ dyne $\mathrm{cm}^{-2}$, showing that, in this case, either the actual loop is quite thin, with $\beta<0.1$, or that the loop is significantly out of equilibrium, perhaps still being filled with evaporating plasma when the decay starts. The magnetic field necessary to confine the flaring plasma is $B_{0}=\sqrt{8 \pi p_{\mathrm{e}}}=200-120 \mathrm{G}$. kG photospheric magnetic fields have been observed through Zeeman splitting in flare stars by e.g. Johns-Krull \& Valenti (1996).

\subsection{The V773 Tau flare}

V773 Tau is a binary weak-line $\mathrm{T}$ Tau star located in the Barnard 209 cloud (in the Tau-Aur star-forming region), at a distance $d \simeq 150 \mathrm{pc}$. The two components have

\footnotetext{
${ }^{2}$ Here, as in the following, the notation $x[x l-x u]$ indicates the best-fit value of the quantity $x$ together with the lower and upper range of the $1 \sigma$ confidence interval, which is typically not symmetric.
} 

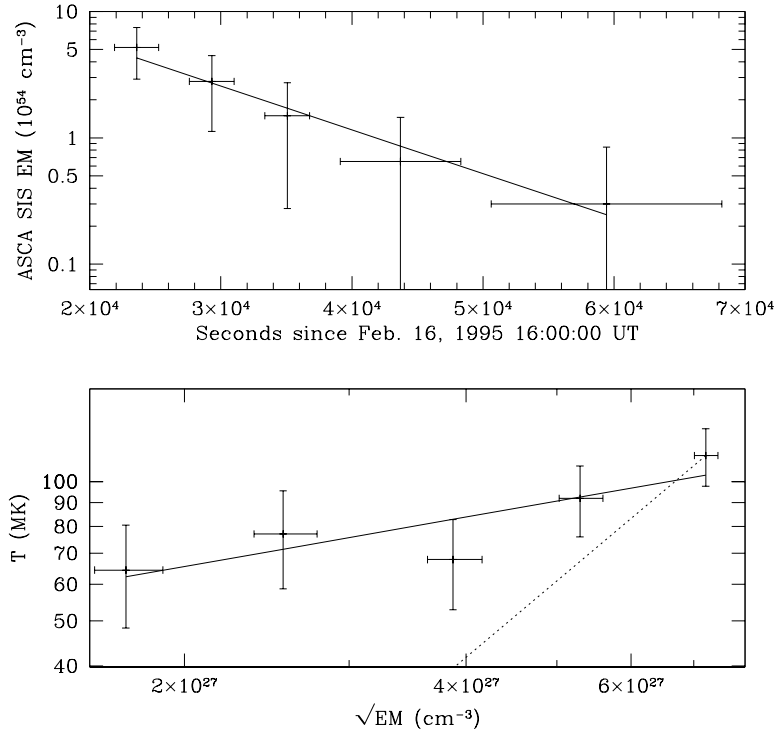

Fig. 2. The evolution of the V773 Tau flare. The top panel shows the flare's emission measure (used as a proxy to the light curve, e.g. to the X-ray count rate), together with the best-fit exponential decay, while the bottom panel shows the evolution of the flare's decay in the $\log T$ vs. $\log n$ plane. The solid line is the best fit to the decay, while the dotted line is the path the decay would have followed if it had been quasi-static with no sustained heating during the decay phase.

spectral types K2 and K5 (Welty 1995), with an estimated radius for either component $R * \simeq 4 R_{\odot}=3 \times 10^{11} \mathrm{~cm}$ (Bouvier et al. 1995). The interbinary separation is $a \simeq$ $5 \times 10^{12} \mathrm{~cm}$ (Welty 1995).

A large X-ray flare detected during an ASCA observation of Barnard 209 has been studied by Tsuboi et al. (1998). The decay time-scale of the flare is $\tau_{\mathrm{LC}}=8.2 \mathrm{ks}$ with a maximum observed temperature in the ASCA-SIS spectrum of $T_{\mathrm{d}} \simeq 110 \mathrm{MK}$ (i.e. a very hot event). The peak X-ray luminosity is $L_{\mathrm{X}} \simeq 10^{33} \mathrm{erg} \mathrm{s}^{-1}$. Tsuboi et al. (1998) note that some of the conditions for the applicability of a quasi-static analysis are actually violated: in particular, the quantity $T^{3.25} / E M$, which is supposed to be constant during the decay, increases toward the end of the event, something which Tsuboi et al. (1998) interpret as evidence for reheating. They then use only the first part of the decay (in which $T^{3.25} / E M$ is approximately constant) to derive the flare parameters. Their quasi-static analysis results in a length of the flaring loop $L \simeq 4 \times 10^{11} \mathrm{~cm} \simeq 1.3 R_{*}$ and a density $n \simeq 3 \times 10^{11} \mathrm{~cm}^{-3}$. The loop is thus derived to be larger than the stars but much smaller than the interbinary separation.

Similarly to the other events discussed here, we have analyzed the event using the hydrodynamic modeling, starting from the spectral analysis results of Tsuboi et al. (1998). The results are shown in Fig. 2: the decay of the temperature during the flare is quite shallow, so that the shape of the light curve is fully dominated by the temporal evolution of the heating, with $\zeta=0.36 \pm 0.12$ and $F(\zeta)=\tau_{\mathrm{LC}} / \tau_{\mathrm{th}}=6.4[5.3-7.8]$. The actual peak temperature of the event is very high, at $T_{\max } \simeq 200 \mathrm{MK}$, and the
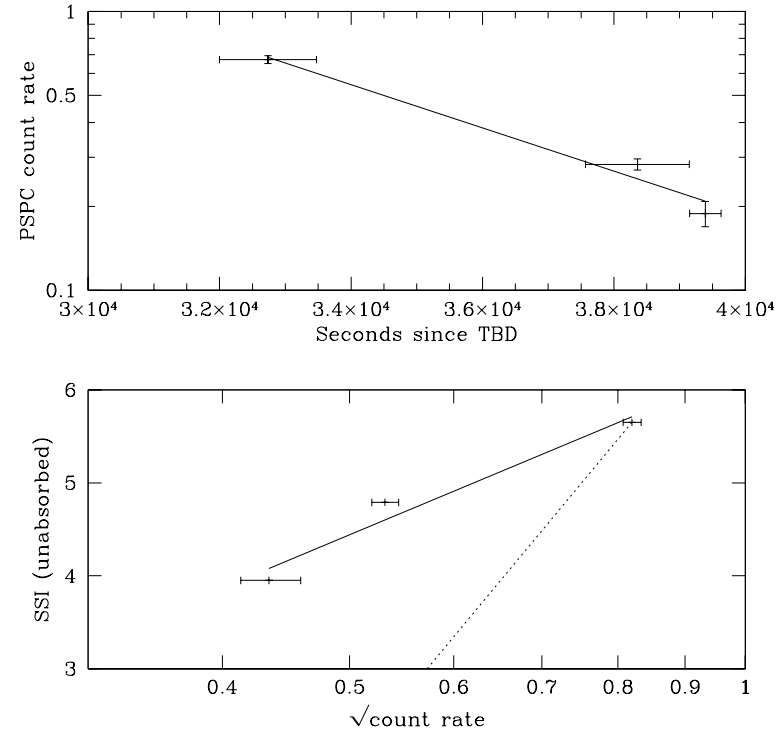

Fig. 3. The evolution of the $\mathrm{LkH} \alpha 92$ flare. The top panel shows the flare's background-subtracted light curve together with the best-fit exponential decay, while the bottom panel shows the evolution of the flare's decay in the SSI (Spectral Shape Index) vs. $\log n$ plane (using the square root of the count rate as a proxy to the density $n$ ). The solid line is the best fit to the decay, while the dotted line is the path the decay would have followed if it had been quasi-static with no sustained heating during the decay phase.

loop length is $L \simeq 7.5[6.1-9.0] \times 10^{10} \mathrm{~cm} \simeq 0.25 \pm 0.05 R_{*}$. The event, notwithstanding its high temperature and $\mathrm{X}$ ray luminosity, is originating in a rather small loop, and therefore close to the stellar surface.

Using the same approach as discussed for YLW 15, the loop volume can be estimated at $V=2.5 \times 10^{31}-2.3 \times$ $10^{32} \mathrm{~cm}^{3}$, and the density and pressure can be derived (using the peak $E M=5.2 \times 10^{55} \mathrm{~cm}^{-3}$ ) at $n_{\mathrm{e}}=1.6 \times 10^{12}$ $5.3 \times 10^{11} \mathrm{~cm}^{-3}$ and $p_{\mathrm{e}}=2 N_{\mathrm{e}} k T_{\max }=8.6 \times 10^{4}-2.9 \times$ $10^{4}$ dyne $\mathrm{cm}^{-2}$. The equilibrium pressure is $p_{\mathrm{RTV}}=3.8 \times$ $10^{4}$ dyne $\mathrm{cm}^{-2}$, compatible with a loop with $\beta \simeq 0.3$. The confining magnetic field is estimated at $B_{0}=1500-850 \mathrm{G}$.

\subsection{The $L k H \alpha 92$ flare}

$\mathrm{LkH} \alpha 92$ is a classical T Tau star in the IC 348 starforming complex, with a $\mathrm{K}$ spectral type, and an estimated radius $R_{*} \simeq 2 R_{\odot}=1.4 \times 10^{11} \mathrm{~cm}$. A large X-ray flare has been detected in its light curve during a ROSAT PSPC observation of IC 348, which has been analyzed by Preibisch et al. (1993) using the quasi-static approach. The analysis of Preibisch et al. (1993) resulted in a decay time-scale of the flare $\tau_{\mathrm{LC}}=7.8 \mathrm{ks}$, and a observed peak temperature determined from the PSPC spectra $T_{\mathrm{d}} \simeq 43 \mathrm{MK}$. The peak X-ray luminosity is $\simeq 5 \times 10^{32} \mathrm{erg} \mathrm{s}^{-1}$. The quasi-static analysis results in a length of the flaring loop $L \simeq 8 \times 10^{10} \mathrm{~cm} \simeq 0.6 R_{*}$ (i.e. a moderate-size loop) and a density $n \simeq 1.5 \times 10^{11} \mathrm{~cm}^{-3}$.

We have analyzed the event using the approach discussed in Reale \& Micela (1998), based on a principal 
component analysis, allowing to make optimal use of the low-resolution, limited bandpass spectra produced by the ROSAT PSPC. The key feature of this approach is the use of a non-parametric temperature indicator (the Spectral Shape Index, or SSI), rather than a parametric fit to the spectrum.

For the analysis, the flare decay has been binned into three intervals, as shown in Fig. 3, extracting backgroundsubtracted spectra for each of them. The quiescent emission (determined in the pre-flare phase) was also subtracted from the flare spectra. The observed decay time scale for the event is $\tau_{\mathrm{LC}}=5.6[4.8-6.8] \mathrm{ks}$. The measured SSI values have been converted to the intrinsic (unabsorbed) SSI values (as discussed in Reale \& Micela 1998) assuming an absorbing column density $N(\mathrm{H})=10^{22} \mathrm{~cm}^{-2}$ (as derived by Preibisch et al. 1993). The slope in the count-rate vs. SSI plot is $\zeta^{\prime}=5.9 \pm 1.2$. The peak SSI corresponds to a maximum temperature for the event $T_{\max }=110 \mathrm{MK}$, and the corresponding loop length is $L=(3.6 \pm 1.1) \times 10^{10} \mathrm{~cm}=(0.26 \pm 0.08) R_{*}$, where the relatively uncertainty is estimated according to the value reported in Table 2 of Reale \& Micela (1998). This flare is therefore confined to a low-lying loop, close to the stellar surface, and with a size falling within the range observed for solar flares. The heating time scale $\tau_{\mathrm{H}}$ is estimated (from Fig. 3 of Reale \& Micela 1998) to be $\tau_{\mathrm{H}} \simeq 2 \times \tau_{\mathrm{LC}}$. The observed flare decay is again dominated by the sustained heating. Note that the peak temperature implied by the peak SSI value is, at $110 \mathrm{MK}$, significantly higher than the maximum temperature measured through spectral fitting (43 MK, see above). This is due to the relatively soft bandpass of the PSPC detector.

Along the same lines discussed above for the YLW 15 event the loop volume can be estimated at $V=2.9 \times 10^{30}$ $2.6 \times 10^{31} \mathrm{~cm}^{3}$ (for $\beta=0.1$ and $\beta=0.3$ respectively), and the density and pressure can be derived (using the peak $\left.E M=4.8 \times 10^{54} \mathrm{~cm}^{-3}\right)$ at $n_{\mathrm{e}}=1.4 \times 10^{12}-4.8 \times 10^{11} \mathrm{~cm}^{-3}$ and $p_{\mathrm{e}}=2 N_{\mathrm{e}} k T_{\max }=4.2 \times 10^{4}-1.4 \times 10^{4} \mathrm{dyne} \mathrm{cm}^{-2}$. The equilibrium pressure is $p_{\mathrm{RTV}}=1.2 \times 10^{4} \mathrm{dyne}_{\mathrm{cm}}{ }^{-2}$, compatible with a $\beta=0.3$ loop near equilibrium (i.e. filled with plasma). The confining magnetic field is estimated at $B_{0}=1000-600 \mathrm{G}$.

\subsection{The HD 283572 flare}

HD 283572 is a relatively nearby WTTS in the Tau-Aur star-forming region, at a (Hipparcos-determined) distance of $128 \mathrm{pc}$. Its optical characteristics are described in detail by Walter et al. (1987), who derived an MK spectral type of G5 IV, and a radius of $3.3 R_{\odot}$, at an assumed distance of $160 \mathrm{pc}$, which becomes $2.7 R_{\odot}=1.9 \times 10^{11} \mathrm{~cm}$ at the Hipparcos distance. Its X-ray characteristics have been studied in detail by Favata et al. (1998). Thanks to its high quiescent $\mathrm{X}$-ray luminosity $\left(L_{\mathrm{X}} \simeq 2 \times 10^{31} \mathrm{erg} \mathrm{s}^{-1}\right)$ it is a well studied object and it has been observed with most X-ray telescopes to date.
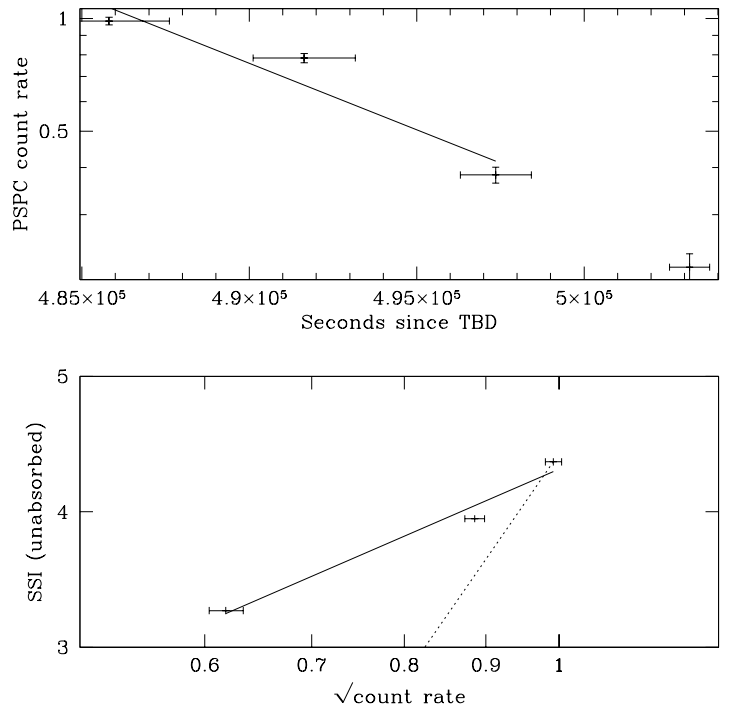

Fig. 4. The evolution of the HD 283572 flare. The top panel shows the flare's background-subtracted light curve together with the best-fit exponential decay, while the bottom panel shows the evolution of the flare's decay in the SSI vs. $\log n$ plane (using the square root of the count rate as a proxy to the density $n$ ). The solid line is the best fit to the decay.

A large flare seen with the ROSAT PSPC is reported (but not discussed in detail) by Stelzer et al. (2000). We have analyzed the event using the same approach based on a principal component analysis as used above for $\mathrm{LkH} \alpha 92$; note that none of the other flares on PMS stars reported by Stelzer et al. (2000) has a sufficient number of events to allow a similar analysis.

The flare decay has been binned into four intervals, as shown in Fig. 4, extracting background-subtracted spectra for each of them and subtracting the quiescent emission from the flare spectra. Only the first three intervals have been used for the analysis, given the very low statistics of the fourth interval. The observed decay time scale for the event is $\tau_{\mathrm{LC}}=12 .[9.4-18.] \mathrm{ks}$; the measured SSI values have been converted to the intrinsic (unabsorbed) SSI values assuming an absorbing column density $N(\mathrm{H})=10^{21} \mathrm{~cm}^{-2}$ (as derived e.g. by Favata et al. 1998). The slope in the count-rate vs. SSI plot of $\zeta^{\prime}=5.1 \pm 0.8$. The peak SSI corresponds to a maximum temperature for the event $T_{\max }=48 \mathrm{MK}$, with a corresponding loop length of $L=(5.0 \pm 1.5) \times 10^{10} \mathrm{~cm}=(0.26 \pm 0.08) R_{*}$. The relatively uncertainty is also in this case is estimated according to the value reported in Table 2 of Reale \& Micela (1998). This flare is therefore also confined in a low-lying loop, close to the stellar surface. The heating time scale $\tau_{\mathrm{H}}$ is estimated (from Fig. 3 of Reale \& Micela 1998) to be $\tau_{\mathrm{H}} \simeq 2 \times \tau_{\mathrm{LC}} \simeq 23.4 \mathrm{ks}$.

The loop volume can be estimated at $V=7.8 \times 10^{30}$ $70 \times 10^{31} \mathrm{~cm}^{3}$ (for $\beta=0.1$ and $\beta=0.3$ respectively), and the density and pressure can be derived (using the peak $\left.E M=4.3 \times 10^{54} \mathrm{~cm}^{-3}\right)$ at $n_{\mathrm{e}}=8.3 \times 10^{11}-2.8 \times 10^{11} \mathrm{~cm}^{-3}$ 
Table 1. A comparison of the physical parameters derived for the flaring events on PMS stars analyzed here. $T_{\mathrm{d}}$ is the maximum temperature measured during the flare (indicated only for the events analyzed through a direct spectral fitting, and absent for the events analyzed with the Spectral Shape Index - SSI - analysis), and $E M$ the maximum emission measure, while $L_{\mathrm{X}}$ is the peak X-ray luminosity. $\tau_{\mathrm{LC}}$ is the decay time for the flare, while $L^{\mathrm{QS}}$ is the length of the flaring loop derived by quasi-static analysis, both in $\mathrm{cm}$ and in units of the stellar radius. $F(\zeta)$ is the ratio $\tau_{\mathrm{LC}} / \tau_{\mathrm{th}}$ between the observed decay time and the natural thermodynamic decay time of the loop (giving a measure of the importance of sustained heating in determining the light curve of the decay). $T_{\max }$ is the actual peak temperature in the flare derived from the observed temperature $T_{\mathrm{d}}$ and the instrument's response or from the value of the SSI, while $L^{\mathrm{HM}}$ is the length of the flaring loop derived through the hydrodynamic modeling approach (again in $\mathrm{cm}$ and in units of the stellar radius). Numerical subscripts indicate the power of 10 by which the relevant quantity has been scaled.

\begin{tabular}{lrrrrrrrrr}
\hline Star & Instr. & $\begin{array}{r}T_{\mathrm{d}} \\
\mathrm{MK}\end{array}$ & $\begin{array}{r}E M_{54} \\
\mathrm{~cm}^{-3}\end{array}$ & $\begin{array}{r}\tau_{\mathrm{LC}} \\
\mathrm{ks}\end{array}$ & $\begin{array}{r}L_{10}^{\mathrm{QS}} \\
\mathrm{cm}\left(R_{*}\right)\end{array}$ & $\begin{array}{r}n_{10}^{\mathrm{QS}} \\
\mathrm{cm}^{-3}\end{array}$ & $\begin{aligned} F(\zeta) \\
T_{\max } \\
\mathrm{MK}\end{aligned}$ & $\begin{array}{r}L_{10}^{\mathrm{HM}} \\
\left.\mathrm{cm}_{*}\right)\end{array}$ \\
\hline YLW 15 & ASCA-GIS & 64 & 15 & 37.9 & $100(3.5)$ & 5 & 2.7 & 150 & $46.0(1.5)$ \\
LkH $\alpha$ 92 & ROSAT-PSPC & 43 & 50 & 5.6 & $8(0.6)$ & 15 & 4.3 & 110 & $3.6(0.3)$ \\
V773 Tau & ASCA-GIS & 110 & 100 & 8.2 & $40(1.3)$ & 30 & 6.4 & 200 & $7.5(0.3)$ \\
HD 283572 & ROSAT-PSPC & - & 4 & 12.0 & - & - & 4.6 & 48 & $5.0(0.3)$ \\
\hline
\end{tabular}

Table 2. The plasma physical parameters derived for the flaring events studied here. For each event, the density $n_{\mathrm{e}}$, the pressure $p$ and the magnetic field $B$ needed to confine the plasma are tabulated under two different assumptions for the loop's aspect (length to diameter) ratio, i.e. $\beta=0.1$ and $\beta=0.3$. Also, the "equilibrium" pressure $p_{\text {RTV }}$ that the plasma would have in a loop satisfying the scaling laws of Rosner et al. (1978) is reported.

\begin{tabular}{lcrrrr}
\hline Star & $\beta$ & $\begin{array}{r}n_{\mathrm{e}} \\
10^{-3} \mathrm{~cm}^{-3}\end{array}$ & $\begin{array}{r}p \\
10^{2} \text { dyne }_{\text { } \mathrm{RmV}^{-2}}\end{array}$ & $\begin{array}{r}B \\
\mathrm{kG}\end{array}$ \\
\hline YLW 15 & 0.1 & 38 & 16 & 27 & 0.2 \\
& 0.3 & 13 & 5 & 27 & 0.1 \\
\hline LkH $\alpha$ 92 & 0.1 & 1400 & 420 & 120 & 1.0 \\
& 0.3 & 480 & 140 & 120 & 0.6 \\
\hline V773 Tau & 0.1 & 1600 & 860 & 380 & 1.5 \\
& 0.3 & 530 & 290 & 380 & 0.9 \\
\hline HD 283572 & 0.1 & 830 & 110 & 8 & 0.5 \\
& 0.3 & 280 & 37 & 8 & 0.3 \\
\hline
\end{tabular}

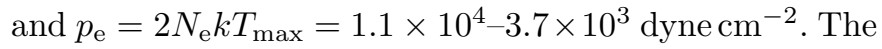
equilibrium pressure is significantly lower, $p_{\mathrm{RTV}}=0.8 \times$ $10^{3}$ dyne $\mathrm{cm}^{-2}$, thus implying either a loop with a very high aspect ratio $\beta>0.3$ (which would however appear unlikely), or to the Spectral Shape Index underestimating the peak temperature of the event: the uncertainty on the SSI derived for a spectrum of about 1000 counts (as it is the case for the hotter spectrum in the HD 283572 event) is, again from Table 2 of Reale \& Micela (1998), $\Delta_{\mathrm{SSI}} \simeq 0.5$, so that the $1 \sigma$ interval for the event's peak temperature is $30-75 \mathrm{MK}$, resulting in a $1 \sigma$ interval for the equilibrium pressure $p_{\mathrm{RTV}}=[0.23 .2] \times 10^{3} \mathrm{dyne}^{-2}$, so that the upper value of the $1 \sigma$ interval is compatible with the $\beta=0.3$ configuration. Also, as shown by the other events analyzed here, large flares on PMS stars tend to be quite hot, so that indeed the nominal peak temperature of $48 \mathrm{MK}$ is lower than expected. The confining magnetic field is estimated at $B_{0}=530-300 \mathrm{G}$.

\section{Discussion}

All flares analyzed in the present paper show evidence for strong sustained heating during the flare decay, so that the decay light curve is dominated by the temporal heating profile and not by the free decay of the heated loop. As a consequence, the decay light curve has limited diagnostic value for the derivation of the physical parameters of the flaring loop, and the quasi-static analysis which has been previously applied to the events discussed here invariably results in loop sizes which are significantly over-estimated. In the presence of such vigorous sustained heating during the decay phase, even the sizes derived by hydrodynamic modeling (which rely on a parameterization of the heating temporal dependence as a decaying exponential) are likely to result in upper limits to the actual loop sizes (as demonstrated by the Algol SAX flare, see Favata 2000 for a discussion). Therefore, the large loop sizes obtained by quasi-static analysis $\left(L \gtrsim 1 R_{*}\right)$ are in reality likely to be significantly smaller, typically by factors $\gtrsim 3$.

This result is in line with the results obtained by applying the same hydrodynamic modeling approach to other classes of active stars, i.e. on Algol (Favata \& Schmitt 1999; Favata et al. 2000b), on flare stars (Reale \& Micela 1998; Favata et al. 2000a; Favata et al. 2000c), on the zeroage main-sequence star AB Dor (Maggio et al. 2000) and on active binaries (Favata 2000). Sustained heating appears to be consistently present during large X-ray flares, so that the flaring regions are much smaller in size than it would be inferred by assuming free decay of their light curve. Therefore the (flaring) corona in very active stars appears to be rather low-lying, close to the photosphere, with no evidence for the very large, extended loops which have been deduced in the past from the quasi-static analysis of large flares. Pre-main sequence stars are no exception to this, as also all flaring events analyzed here appear to be due to low-lying structures.

As discussed by Favata et al. (2000b), the location of large flaring events appears in general to be compatible with their being located at high stellar latitude, close to 
the stellar poles, with no evidence for low-latitude, nearequatorial structures (as for example typically present on the Sun). No large flare has been observed to self-eclipse even when its duration is comparable to the stellar rotational period; given the small size of the flaring regions, the polar region is thus the only plausible location for the flaring plasma. For the events discussed here, the ones on LkH $\alpha$ 92, V773 Tau and HD 283572 have durations significantly shorter than the plausible rotational period of the stars and therefore this line of reasoning cannot be used to argue for a polar location of the flaring region. For the event on YLW 15, Montmerle et al. (2000) argue that the object is a fast rotator, with a period $P_{\text {rot }}=16-20 \mathrm{hr}$. All three events reported by Tsuboi et al. (2000) have a duration of $\simeq 20 \mathrm{hr}$ each, and in no case there is evidence for self-eclipse. Given the relatively small size derived here for the flaring region, a polar location for the flaring region on YLW 15 appears strongly favored.

This scenario (a low-lying flaring loop, likely located on the polar region of the star) is not compatible with the explanation for the large flares on YLW 15 proposed by Montmerle et al. (2000), who, on the basis of the long loop size derived from the quasi-static analysis argue for a flaring loop with one foot anchored on the stellar surface and the other onto the (edge of the) accretion disk. The smaller loop size for the YLW 15 flare implied by the hydrodynamic modeling discussed in the present paper makes such loops implausible, and therefore it also casts a doubt on the framework used by Montmerle et al. (2000) to explain the repeated flaring events seen on YLW 15 (i.e. the stressing of magnetic field lines spanning both the star and the disc).

A summary of the characteristics of the event for each of the flares studied here is reported in Table 1, and a summary of the resulting plasma parameters in Table 2. For the events on the CCTS LkH $\alpha 92$ and on the WTTS V773 Tau and HD 283572 the resulting loop sizes are consistently around $L \simeq 0.3 R_{*}$. The event on the protostar YLW 15 is somewhat larger, with a nominal length $L \simeq 1.5 R_{*}$. However, the pressure derived even assuming $\beta=0.1$ is significantly lower than the equilibrium pressure derived from the Rosner et al. (1978) scaling laws. This points toward the loop being far from equilibrium and only partially filled with hot plasma, so that the procedure used here is likely to over-estimate the actual loop size. Both these elements make us consider the length estimate derived here as an upper limit to the actual size of the flaring region. Therefore the characteristics of the X-ray flare observed on YLW 15 appear to be similar to the ones observed on older stars, so that there may not be any need to invocate a separate mechanism to explain the observed behavior. Whether flares on protostars are in general more "out of equilibrium" than flares on older stars is of course impossible to say on the basis of a single event, and clearly study of a significant number of flaring events will be needed to assess whether any such general difference exists.

Acknowledgements. FR, GM acknowledge the partial support of ASI and MURST.

\section{References}

Bouvier, J., Covino, E., Kovo, O., et al. 1995, A\&A, 299, 89 Favata, F. 2000, in ASP Conf. Ser., Vol. in press, Proc. of X-ray Astronomy 2000, Palermo Sep. 2000, ed. R. Giacconi, L. Stella, \& S. Serio, in press [http://xxx.lanl.gov/abs/astro-ph/0011485]

Favata, F., Micela, G., \& Reale, F. 2000a, A\&A, 354, 1021

Favata, F., Micela, G., Reale, F., Sciortino, S., \& Schmitt, J. H. M. M. 2000b, A\&A, 362, 628

Favata, F., Micela, G., \& Sciortino, S. 1998, A\&A, 337, 413

Favata, F., Reale, F., Micela, G., et al. 2000c, A\&A, 353, 987

Favata, F., \& Schmitt, J. H. M. M. 1999, A\&A, 350, 900

Feigelson, E. D., \& Montmerle, T. 1999, ARA\&A, 37, 363

Glassgold, A. E., Feigelson, E. D., \& Montmerle, T. 2000, in Protostars and Planets IV, ed. V. Mannings, A. P. Boss, \& S. S. Russell (Tucson: University of Arizona Press) 429, 429

Grosso, N., Montmerle, T., Feigelson, E. D., et al. 1997, Nature, 387,56

Hartmann, L. 1998, Accretion processes in star formation (Cambridge University Press)

Johns-Krull, C. M., \& Valenti, J. A. 1996, ApJ, 459, L95

Maggio, A., Pallavicini, R., Reale, F., \& Tagliaferri, G. 2000, A\&A, 356, 627

Montmerle, T., Grosso, N., Tsuboi, Y., \& Koyama, K. 2000, ApJ, 529, in press

Preibisch, T., Zinnecker, H., \& Schmitt, J. H. M. M. 1993, A\&A, 279, L33

Reale, F., Betta, R., Peres, G., Serio, S., \& McTiernan, J. 1997, A\&A, 325, 782

Reale, F., \& Micela, G. 1998, A\&A, 334, 1028

Rosner, R., Tucker, W. H., \& Vaiana, G. S. 1978, ApJ, 220, 643

Schmitt, J. H. M. M., \& Favata, F. 1999, Nature, 401, 44

Serio, S., Reale, F., Jakimiec, J., Sylwester, B., \& Sylwester, J. 1991, A\&A, 241, 197

Stelzer, B., Neuhäuser, R., \& Hambaryan, V. 2000, A\&A, 356, 949

Tsuboi, Y., Imanishi, K., Koyama, K., Grosso, N., \& Montmerle, T. 2000, ApJ, 532, 1089

Tsuboi, Y., Koyama, K., Murakami, H., et al. 1998, ApJ, 503, 894

van den Oord, G. H. J., \& Mewe, R. 1989, A\&A, 213, 245

Walter, F. M., Brown, A., Linsky, J. L., et al. 1987, ApJ, 314, 297

Welty, A. D. 1995, AJ, 110, 776 